\title{
How can we make oral nutritional supplementation in the community more appropriate and cost-effective?
}

\author{
V. E. Pout \\ Department of Nutrition and Dietetics, William Harvey Hospital, Willesborough, Ashford, Kent TN24 OLZ, UK
}

Malnutrition is a frequently unrecognised disease state ${ }^{(1)}$ costing the UK an estimated $£ 13$ billion in $2009^{(2)}$. A food-first approach to malnutrition should be taken in the community and oral nutritional supplements (ONS) prescribed appropriately to ensure effective use of healthcare resources ${ }^{(1)}$. This project explored factors influencing effective ONS prescription.

A large GP surgery provided lists of patients receiving ONS in March $(n=48)$ and July $(n=42) 2008$. Reasons for the cessation of ONS prescription in the interim time were recorded. Current ONS patients had data collected regarding demographics, reasons for initiation/cessation of ONS prescription, ONS prescription initiator and duration, anthropometric measurements and dietetic involvement.

Those patients not receiving dietetic intervention were discussed with the practice manager and community nurses to devise an appropriate follow-up plan.

The demographics collected illustrated that the age distribution was skewed towards those aged over 65 years. For $57 \%$ of patients, no reason for initiation of ONS was given in the GP notes. The most common reasons when available were poor intake (39\%) and swallowing problems (12\%). The most common reason for the cessation of ONS prescription between March and July 2008 was death (32\%). Anthropometric data were found to be poorly recorded with $12 \%$ having no weight and $35 \%$ not having a recorded weight within the last 6 months. Forty-two percent of patients had been receiving ONS for more than 1 year with the most common initiator being the GP $(61 \%)$. There was no dietetic involvement in $42 \%$ of patients.

Education regarding nutritional screening and appropriate prescription of ONS had been provided to the General Practitioners in the surgery in Autumn 2007. This was repeated for the Community Nurses in Spring 2008. Post training 34\% of patients had their prescriptions reviewed and stopped and $8 \%$ had their prescriptions changed to a more cost effective product. In total, $19 \%$ were referred to Dietetics for supported cessation of oral nutritional supplements.

Discussions initiated by this study regarding patients not receiving dietetic intervention highlighted the importance of multidisciplinary team-working in devising appropriate follow-up plans.

Through discussion it was evident that the above training had a noticeable effect on the use of ONS. Training resulted in review of ONS prescription in long-term patients thereby providing the opportunity for cost-effective prescribing. Savings as a consequence of this training project have been estimated at $£ 12,464$ in 2009 for this surgery.

1. National Institute for Health and Clinical Excellence (2006) Nutrition Support in Adults: Oral Nutrition Support, Enteral Tube Feeding and Parenteral Nutrition. London: National Collaborating Centre for Acute Care.

2. British Association of Parenteral and Enteral Nutrition (2009) Combating Malnutrition-Recommendations for Action. Redditch: The British Association for Parenteral and Enteral Nutrition. 